International Journal of Trend in Scientific Research and Development (IJTSRD)

Volume: 3 | Issue: 3 | Mar-Apr 2019 Available Online: www.ijtsrd.com e-ISSN: 2456 - 6470

\title{
The Estimation for the Eigenvalue of Quaternion Doubly Stochastic Matrices using Gerschgorin Balls
}

\author{
Dr. Gunasekaran $K^{1}$, Mrs. Seethadevi R ${ }^{2}$ \\ ${ }^{1}$ Head and Associative Professor, ${ }^{2} \mathrm{PhD}$ Scholar \\ ${ }^{1,2}$ Department of Mathematics, Government Arts College (Autonomous), Kumbakonam, Tamil Nadu, India
}

\begin{abstract}
How to cite this paper: Dr. Gunasekaran K | Mrs. Seethadevi R "The Estimation for the Eigenvalue of Quaternion Doubly Stochastic Matrices using Gerschgorin Balls" Published in International Journal of Trend in Scientific Research and Development (ijtsrd), ISSN: 24566470, Volume-3 | Issue-3, April 2019, pp.667-670, URL: http://www.ijtsrd.co $\mathrm{m} /$ papers/ijtsrd228 60.pdf

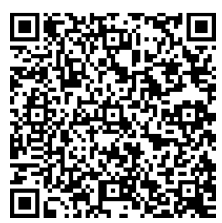
IITSRD22860

Copyright (C) 2019 by author(s) and International Journal of Trend in Scientific Research and Development Journal. This is an Open Access article distributed under the terms of the Creative Commons

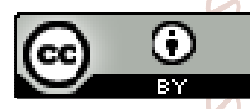
Attribution License (CC BY 4.0) (http://creativecommons.org/licenses/ by $/ 4.0$ )
\end{abstract}

\section{INTRODUCTION}

In the past two decades due to study on matrix theory and some engineering background problems, many scholars dedicated to special matrix, and obtained some important and valuable results. Hing zhu-2007 yigeng Huang-1994). But in combination matrix theory, combinatorics, probability theorey. Mathematical economics and reliability theory etc., area there is a special class of non-negative quaternion doubly stochastic matrix, which in recent years becomes concerned.

The article discuss location, distribution and estimate of the eigen value for quaternion doubly stochastic matrix section 2 introduces the concept of quaternion doubly stochastic matrix and generalized quaternion doubly stochastic matrix. Section 3 gives a few estimation theorems of quaternion doubly stochastic matrix eigen value, also the eigen value distribution for tensor Product of two quaternion doubly stochastic matrices is obtained. In section 4 we discuss the eigen value distribution for generalized quaternion doubly stochastic matrices, (3) eigen value estimate of quaternion doubly stochastic matrix.

\section{Definition 1}

If both $\mathrm{A}$ and $\mathrm{A}^{\mathrm{T}}$ are quaternion row stochastic matrices, $\mathrm{A}$ is quaternion double stochastic matrix; Row stochastic matrix, column stochastic matrix and quaternion double stochastic matrix are called stochastic matrix, denoted by $\mathrm{S}(\mathrm{n})$.

\section{Definition 2}

The first generalized quaternion row stochastic matrix, the first generalized quaternion column stochastic matrix and the first generalized quaternion double stochastic matrix are called the first generalized quaternion stochastic matrix, denoted by $S_{I}(n)$.

\section{Definition 3}

The second generalized quaternion row stochastic matrix, the second generalized quaternion column stochastic matrix and the second generalized quaternion double stochastic matrix are called the second generalized quaternion stochastic matrix, denoted by $\mathrm{S}_{\text {II }}(\mathrm{n})$.

\section{Definition 4}

The third generalized quaternion row stochastic matrix, the third generalized quaternion column stochastic matrix and the third generalized quaternion double stochastic matrix 
International Journal of Trend in Scientific Research and Development (IJTSRD) @ www.ijtsrd.com eISSN: 2456-6470

are called the third generalized quaternion stochastic matrix, denoted by $S_{\text {III }}(n)$.

$\mathrm{S}_{\mathrm{I}}(\mathrm{n}), \mathrm{S}_{\mathrm{II}}(\mathrm{n}), \mathrm{S}_{\mathrm{III}}(\mathrm{n})$ are called generalized stochastic matrices obviously for $S(n), S_{I}(n), S_{I I}(n)$ and $S_{\text {III }}(n)$ we have the following simple conclusions

1. $\mathrm{S}(\mathrm{n}) \subset \mathrm{S}_{\mathrm{I}}(\mathrm{n}) \subset \mathrm{S}_{\mathrm{III}}(\mathrm{n})$

2. $\mathrm{S}(\mathrm{n}) \subset \mathrm{S}_{\mathrm{II}}(\mathrm{n}) \subset \mathrm{S}_{\mathrm{III}}(\mathrm{n})$

3. $\mathrm{S}(\mathrm{n}) \subset \mathrm{S}_{\mathrm{II}}(\mathrm{n}) \subset \mathrm{S}_{\mathrm{III}}(\mathrm{n})$

\section{Theorem 1}

Suppose $A=\left(a_{c d}\right)_{n \times n}$ is a quaternion doubly stochastic matrix and $\mathrm{m}=\min \left\{\left|\mathrm{a}_{\mathrm{cc}}\right|, \mathrm{c}, \mathrm{d}=1,2, \ldots, \mathrm{n}\right\}$ then $\lambda(A) \subset G(A)=\{Z:|Z-m| \leq 1-m\}$. Where $\lambda(A)$ is denoted the whole eigen values of matrix $A, G(A)$ is gerschgorin balls of matrix $\mathrm{A}$.

\section{Proof:}

Since $\lambda$ is an arbitrary eigen value of matrix $\mathrm{A}=\left(\mathrm{a}_{\mathrm{cd}}\right)_{\mathrm{n} \times \mathrm{n}}$ and $\mathrm{X}=\left(\mathrm{x}_{1}, \mathrm{x}_{2}, \ldots, \mathrm{x}_{\mathrm{n}}\right)^{\mathrm{T}} \in \mathrm{H}^{\mathrm{n} \times 1}$ is the corresponding column eigen vector, lin let $\mathrm{y}_{\mathrm{c}}=\frac{\mathrm{x}_{\mathrm{c}}}{\mathrm{t}_{\mathrm{c}}}$ where $_{\mathrm{c}}(\mathrm{c}=1,2, \ldots, \mathrm{n})$,

$$
\begin{gathered}
\left|\lambda \mathrm{t}_{\mathrm{e}}-\mathrm{a}_{\mathrm{ee}}\right| \leq \mathrm{Q}_{\mathrm{e}}=\sum_{\substack{\mathrm{d}=1 \\
\mathrm{~d} \neq \mathrm{e}}}^{\mathrm{n}}\left|\mathrm{a}_{\mathrm{cd}}\right| \\
=1-\mathrm{a}_{\mathrm{ee}}
\end{gathered}
$$

Therefore,

$|\lambda-\mathrm{e}|=\left|\lambda-\mathrm{a}_{\mathrm{ee}}+\mathrm{a}_{\mathrm{ee}}-\mathrm{e}\right| \leq\left|\lambda-\mathrm{a}_{\mathrm{ee}}\right|+\left|\mathrm{a}_{\mathrm{ee}}-\mathrm{e}\right| \leq 1-\mathrm{a}_{\mathrm{ee}}+\mathrm{a}_{\mathrm{ee}}-\mathrm{e}=1-\mathrm{e}$

Since $\lambda$ is an arbitrary eigen value of quaternion doubly stochastic matrix $\mathrm{A}=\left(\mathrm{a}_{\mathrm{cd}}\right)_{\mathrm{n} \times \mathrm{n}}$,

then

$\lambda(A) \subset G(A)=\{Z:|Z-m| \leq 1-m\}$.

So the eigen values of $\mathrm{A}$ are located in the gerschgorin balls whose center $\mathrm{m}=\min \left\{\mathrm{a}_{\mathrm{cc}}, \mathrm{c}, \mathrm{d}=1,2, \ldots, \mathrm{n}\right\}$ and radius $1-\mathrm{m}$

Hence proved.

\section{Theorem 2}

Suppose $A=\left(a_{c d}\right)_{n \times n}$ is a quaternion doubly stochastic matrix and

$$
\mathbf{M}_{\mathrm{cd}}=\max _{\mathrm{cd}}\left\{\left|\mathrm{a}_{\mathrm{cd}}\right|, \mathrm{c}, \mathrm{d}=1,2, \ldots, \mathrm{n}\right\} \text { then }
$$

$$
\lambda(\mathrm{A}) \subset \mathrm{G}(\mathrm{A})=\left\{\mathrm{Z}:\left|\mathrm{Z}-\frac{\operatorname{Tra}(\mathrm{A})}{\mathrm{n}}\right| \leq \sqrt{\frac{\mathrm{n}-1}{\mathrm{n}}\left(\sum_{\mathrm{d}=1}^{\mathrm{n}} \sum_{\mathrm{c}=1}^{\mathrm{n}} \mathrm{M}_{\mathrm{cd}}\right)-\frac{(\operatorname{Tra}(\mathrm{A}))^{2}}{\mathrm{n}}}\right\}
$$

Where $\lambda(A)$ is denoted the whole eigen values of matrix $\left|y_{c}\right|=\max \left|y_{c}\right|(c=1,2, \ldots, n)$ and from $A x=\lambda x$

$$
\begin{gathered}
\lambda y_{c} t_{c}=\sum_{d=1}^{n} a_{c d} t_{d} y_{d} \\
\lambda y_{e} t_{e}=\sum_{\substack{d=1 \\
d \neq e}}^{n} a_{c d} t_{d} y_{d} \\
\lambda y_{e} t_{e}=a_{e e} t_{e} y_{e}+\sum_{\substack{d=1 \\
d \neq e}}^{n} a_{c d} t_{d} y_{d}
\end{gathered}
$$

A. $G(A)$ is denoted balls whose center is $\frac{\operatorname{Tra}(A)}{n}$ and radius

$\lambda(A) \subset G(A)=\left\{Z:\left|Z-\frac{\operatorname{Tra}(A)}{n}\right| \leq \sqrt{\frac{n-1}{n}\left(\sum_{d=1}^{n} \sum_{c=1}^{n} M_{c d}\right)-\frac{(\operatorname{Tra}(A))^{2}}{n}}\right\}$ is

Proof:

From paper (yixiGu, 1994) and for arbitrary matrix A

$\left|\lambda-\frac{\operatorname{Tra}(\mathrm{A})}{\mathrm{n}}\right| \leq \sqrt{\frac{\mathrm{n}-1}{\mathrm{n}}\left(\|\mathrm{A}\|_{\mathrm{F}}^{2}\right)-\frac{(\operatorname{Tra}(\mathrm{A}))^{2}}{\mathrm{n}}}$ and because

Multiply right each item of the above equation with $\mathrm{y}_{\mathrm{e}}{ }^{*}$

$$
\begin{gathered}
\lambda y_{e} t_{e} y_{e}^{*}=a_{e e} t_{e} y_{e} y_{e}^{*}+\sum_{\substack{d=1 \\
d \neq e}}^{n} a_{c d} t_{d} y_{d}^{*} y_{e} \\
\lambda t_{e} y_{e} y_{e}^{*}=a_{e e} t_{e} y_{e} y_{e}^{*}+\sum_{\substack{d=1 \\
d \neq e}}^{n} a_{c d} t_{d} y_{d}^{*} y_{e} \\
\lambda t_{e} y_{e} y_{e}^{*}=a_{e e} t_{e} y_{e} y_{e}^{*}+\sum_{\substack{d=1 \\
d \neq e}}^{n} a_{c d} t_{d} y_{d}^{*} y_{e} \\
\left(\lambda t_{e}-a_{e e} t_{e}\right)=\sum_{\substack{d=1 \\
d \neq e}}^{n} \frac{t_{d} a_{e d} y_{d} y_{e}^{*}}{\left|y_{e}\right|^{2}}
\end{gathered}
$$

$A=\left(a_{c d}\right)_{n \times n} \in H(n),\|A\|_{F}^{2} \leq \sum_{c=1}^{n} M_{c d} \sum_{d=1}^{n} M_{c d}$. So we have $\lambda(A) \subset G(A)=\left\{Z:\left|Z-\frac{\operatorname{Tra}(A)}{n}\right| \leq \sqrt{\frac{n-1}{n} \sum_{c=1}^{n} \sum_{d=1}^{n} M_{c d}-\frac{(\operatorname{Tra}(A))^{2}}{n}}\right\}$

Similarly we get,

$$
\lambda(\mathrm{A}) \subset \mathrm{G}(\mathrm{A})=\left\{\mathrm{Z}:\left|\mathrm{Z}-\frac{\operatorname{Tra}(\mathrm{A})}{\mathrm{n}}\right| \leq \sqrt{\frac{\mathrm{n}-1}{\mathrm{n}}\left(1-\frac{(\operatorname{Tra}(\mathrm{A}))^{2}}{\mathrm{n}}\right)}\right\}
$$

This completes the proof of the theorem.

\section{Theorem 3}

Suppose $A=\left(a_{c d}\right)_{n \times n}$ and $B=\left(b_{c d}\right)_{m \times m}$ are quaternion doubly stochastic matrices, $\mathrm{m}_{1}=\min \left\{\left|\mathrm{a}_{\mathrm{cc}}\right|, \mathrm{c}=1,2, . ., \mathrm{n}\right\}$ and radius is $1-\mathrm{m}_{1}$, and Gerschgorine balls whose center is

$$
\left|\lambda \mathrm{t}_{\mathrm{e}}-\mathrm{a}_{\mathrm{ee}} \mathrm{t}_{\mathrm{e}}\right| \leq \sum_{\substack{\mathrm{d}=1 \\ \mathrm{~d} \neq \mathrm{e}}}^{\mathrm{n}}\left|\mathrm{t}_{\mathrm{d}} \mathrm{a}_{\mathrm{cd}}\right|
$$




$$
\begin{aligned}
& \mathrm{m}_{2}=\min \left\{\left|\mathrm{a}_{\mathrm{dd}}\right|, \mathrm{d}=1,2, \ldots, \mathrm{m}\right\} \quad \text { and radius is } \\
& \lambda(\mathrm{A}) \subset \mathrm{G}(\mathrm{A})=\bigcup_{\mathrm{c}, \mathrm{d}=1}^{\mathrm{n}}\left\{\mathrm{Z} ;\left|\mathrm{Z}-\mathrm{a}_{\mathrm{cc}}\right| \leq \sqrt{(\mathrm{n}-1)} \mathrm{M}_{1}\left(1-\mathrm{a}_{\mathrm{cc}}\right)\right\} .
\end{aligned}
$$

\section{Proof:}

Let $\lambda(A)=\left\{\lambda_{1}, \lambda_{2}, \ldots, \lambda_{n}\right\}$ and $\lambda(B)=\left\{\mu_{1}, \mu_{2}, \ldots, \mu_{n}\right\}$, $\lambda(\mathrm{A}) \subset\left\{\mathrm{Z}:\left|\mathrm{Z}-\mathrm{m}_{1}\right| \leq 1-\mathrm{m}_{1}\right\}$

$\lambda(\mathrm{A}) \subset\left\{\mathrm{Z}:\left|\mathrm{Z}-\mathrm{m}_{2}\right| \leq 1-\mathrm{m}_{2}\right\} \quad$ and $\quad$ since

$\lambda(\mathrm{A} \otimes \mathrm{B})=\left[\lambda_{\mathrm{c}} \mu_{\mathrm{d}}\right] \mathrm{c}=1,2, \ldots, \mathrm{n}, \mathrm{d}=1,2, \ldots, \mathrm{n}$.

Therefore, the eigen values of tensor product for matrix $A$ and Matrix $B$ are located in the oval region $G(A \otimes B)$. Hence proved.

\section{Theorem 4}

Suppose $\mathrm{A}=\left(\mathrm{a}_{\mathrm{cd}}\right)_{\mathrm{n} \times \mathrm{n}}$ is quaternion doubly stochastic matrix $\quad$ and $M_{1}=\max _{c d}\left\{\left|a_{c d}\right|, c, d=1,2, \ldots, n\right\}$, $\lambda(A) \subset G(A)=\bigcup_{c, d=1}^{n}\left\{Z ;\left|Z-a_{c c}\right| \leq \sqrt{(n-1) M_{1}\left(1-a_{c c}\right)}\right\}$ where $\lambda(A)$ is denoted the whole eigen values of matrix $A$, $\mathrm{G}(\mathrm{A})$ is denoted the generalized Greschgorin balls of matrix A.

\section{Proof:}

Because $\lambda$ is an arbitrary eigen value of matrix $\mathrm{A}=\left(\mathrm{a}_{\mathrm{cd}}\right)_{\mathrm{n} \times \mathrm{n}}$ and $\mathrm{X}=\left(\mathrm{x}_{1}, \mathrm{x}_{2}, \ldots, \mathrm{x}_{\mathrm{n}}\right)^{\mathrm{T}} \in \mathrm{H}^{\mathrm{n} \times 1}$ is $\mathrm{D}$ the corresponding column eigenvector for $A x=\lambda x$, we set

$$
\begin{gathered}
\text { So, } \sum_{\substack{\mathrm{d}=1 \\
\mathrm{~d} \neq \mathrm{e}}}^{\mathrm{n}} \mathrm{a}_{\mathrm{ed}} \mathrm{x}_{\mathrm{d}}=\lambda_{\mathrm{xe}} \\
\left(\lambda-\mathrm{a}_{\mathrm{ee}}\right) \mathrm{x}_{\mathrm{e}}=\sum_{\substack{\mathrm{d}=1 \\
\mathrm{~d} \neq \mathrm{e}}}^{\mathrm{n}} \mathrm{a}_{\mathrm{ed}} \mathrm{x}_{\mathrm{d}}
\end{gathered}
$$$$
\begin{aligned}
& \text { Internati Trer } \\
& \text { of matrix } \\
& { }_{\mathrm{x} \times 1} \text {, we set }
\end{aligned}
$$

From schwarz inequality $\mathrm{n}$ triagonal inequality, we have the following result.

$$
\begin{aligned}
& \left|\lambda-\mathrm{a}_{\mathrm{ee}}\right|=\left|\frac{\sum_{\mathrm{d} \neq \mathrm{e}} \mathrm{a}_{\mathrm{ed}} \mathrm{x}_{\mathrm{d}} \mathrm{x}_{\mathrm{e}}^{*}}{\left|\mathrm{x}_{\mathrm{e}}^{2}\right|}\right| \leq \sqrt{\sum_{\mathrm{d} \neq \mathrm{e}}\left|\mathrm{a}_{\mathrm{ed}}\right|^{2}} \sqrt{\sum_{\mathrm{d} \neq \mathrm{e}}\left|\frac{\mathrm{x}_{\mathrm{d}}}{\left|\mathrm{x}_{\mathrm{e}}\right|}\right|^{2}}
\end{aligned}
$$

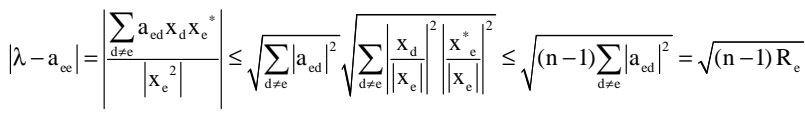

$$
\begin{aligned}
& \text { Where } \mathrm{R}_{\mathrm{e}}=\sqrt{\sum_{\mathrm{d} \neq \mathrm{e}}\left|\mathrm{a}_{\mathrm{ed}}\right|^{2}}, \mathrm{e}=1,2, \ldots, \mathrm{n} \text { and since } \\
& \mathrm{R}_{\mathrm{e}}=\sqrt{\sum_{\mathrm{d} \neq \mathrm{e}}\left|\mathrm{a}_{\mathrm{ed}}\right|^{2}} \leq \sqrt{\mathrm{M}_{\mathrm{e}} \sum_{\mathrm{d} \neq \mathrm{e}}\left|\mathrm{a}_{\mathrm{ed}}\right|}=\sqrt{\mathrm{M}_{\mathrm{e}}\left(1-\mathrm{a}_{\mathrm{ee}}\right)}, \\
& \mathrm{e}=1,2, \ldots, \mathrm{n},\left|\lambda-\mathrm{a}_{\mathrm{ee}}\right| \leq \sqrt{(\mathrm{n}-1) \mathrm{M}_{\mathrm{e}}\left(1-\mathrm{a}_{\mathrm{ee}}\right)}
\end{aligned}
$$

holdsbecause $\lambda$ is an arbitrary eigenvalue of a matrix $A$.

$$
\begin{gathered}
\lambda(\mathrm{A}) \subset \mathrm{G}(\mathrm{A})=\bigcup_{\mathrm{c}, \mathrm{d}=1}^{\mathrm{n}}\left\{\mathrm{Z} ;\left|\mathrm{Z}-\mathrm{a}_{\mathrm{cc}}\right| \leq \sqrt{(\mathrm{n}-1) \mathrm{M}_{1}\left(1-\mathrm{a}_{\mathrm{cc}}\right)}\right\} \\
\text { The theorem is proven. }
\end{gathered}
$$

Eigen value estimate for generalize quaternion doubly stochastic matrix.

Theorem 5: (yuanlu, 2010)

Suppose $A=\left(a_{c d}\right)_{n \times n} \in S_{\text {III }}(n), a_{c c}$ and $a_{d d}$ are the most small diagonal elements in $\mathrm{A}$, then

$$
\lambda(\mathrm{A}) \subset \mathrm{G}(\mathrm{A})=\left\{\mathrm{Z}:\left|\mathrm{Z}-\mathrm{a}_{\mathrm{cc}}\right|\left|\mathrm{Z}-\mathrm{a}_{\mathrm{dd}}\right| \leq\left(\mathrm{S}-\mathrm{a}_{\mathrm{cc}}\right)\left(\mathrm{S}-\mathrm{a}_{\mathrm{dd}}\right)\right\}
$$

Where $\lambda(\mathrm{A})$ is denoted the whole eigen values of matrix $A, G(A)$ is denoted cassini oval region of matrix $A$.

Theorem 6: (yancheng, 2010)

Suppose $\quad A=\left(a_{\text {cd }}\right)_{\mathrm{n} \times \mathrm{n}} \in \mathrm{S}_{\mathrm{III}}(\mathrm{n}) \quad$ and $\mathrm{B}=\left(\mathrm{b}_{\mathrm{cd}}\right)_{\mathrm{m} \times \mathrm{m}} \in \mathrm{S}_{\mathrm{III}}(\mathrm{m})$ are quaternion doubly stochastic matrices

$\lambda(\mathrm{A} \otimes \mathrm{B}) \subset \mathrm{G}(\mathrm{A} \otimes \mathrm{B})=\left\{\mathrm{Z}:\left|\mathrm{Z}-\mathrm{a}_{\mathrm{cc}}\right|\left|\mathrm{Z}-\mathrm{a}_{\mathrm{dd}}\right| \leq\left(\mathrm{S}-\mathrm{a}_{\mathrm{cc}}\right)\left(\mathrm{S}-\mathrm{a}_{\mathrm{dd}}\right)\right\}$

$$
\left\{\mathrm{Z}:\left|\mathrm{Z}-\mathrm{b}_{\mathrm{cc}}\right|\left|\mathrm{Z}-\mathrm{b}_{\mathrm{dd}}\right| \leq\left(\mathrm{S}-\mathrm{b}_{\mathrm{cc}}\right)\left(\mathrm{S}-\mathrm{b}_{\mathrm{dd}}\right)\right\}
$$

Where $\lambda(A \otimes B)$ is denoted the whole eigen values of tensor product for matrix $A$ and matrix $B, G(A \otimes B)$ is the oval region of the product for cassini oval region elements of matrix $\mathrm{A}$ and cassini oval region elements of matrix B

\section{Proof:}

This proof is same to theorem(3) which is leaven for readers

\section{Theorem 7:}

$$
\begin{aligned}
& \text { Suppose } A=\left(a_{c d}\right)_{n \times n} \in S_{I I I}(n) \text { and } \\
& m=\min \left\{\left|a_{c c}\right|, c=1,2, \ldots, n\right\}, \text { then } \\
& \lambda(A) \subset G(A)=\{Z:|Z-m| \leq S+e\}
\end{aligned}
$$

Where $\lambda(\mathrm{A})$ is denoted the wholeeigen values of matrix A , $G(A)$ is the balls whose center is $\mathrm{m}=\min \left\{\left|\mathrm{a}_{\mathrm{cc}}\right|, \mathrm{c}=1,2, \ldots, \mathrm{n}\right\}$ and radius $\mathrm{S}+\mathrm{e}$

Proof:

From Gerschgorin balls theorem, we have

$$
\left|\lambda-\mathrm{a}_{\mathrm{ee}}\right| \leq \mathrm{Q}_{\mathrm{e}}=\sum_{\mathrm{d}=1}^{\mathrm{n}}\left|\mathrm{a}_{\mathrm{ed}}\right|=\mathrm{S}-\left|\mathrm{a}_{\mathrm{ee}}\right|
$$

Therefore,

$|\lambda-\mathrm{e}|=\left|\lambda-\mathrm{a}_{\text {ee }}+\mathrm{a}_{\text {ee }}-\mathrm{e}\right| \leq\left|\lambda-\mathrm{a}_{\text {ee }}\right|+\left|\mathrm{a}_{\mathrm{ee}}-\mathrm{e}\right| \leq \mathrm{S}-\left|\mathrm{a}_{\mathrm{ee}}\right|+\left|\mathrm{a}_{\mathrm{ee}}\right|+\mathrm{e}=\mathrm{S}+\mathrm{e}$

Because $\lambda$ is an arbitrary eigen value of matrix $\mathrm{A}=\left(\mathrm{a}_{\mathrm{cd}}\right)_{\mathrm{n} \times \mathrm{n}}$

$$
\lambda(\mathrm{A}) \subset \mathrm{G}(\mathrm{A})=\{\mathrm{Z}:|\mathrm{Z}-\mathrm{e}| \leq 1+\mathrm{e}\}
$$

So the eigen values of matrix $\mathrm{A}$ are located in the disc whose center is $\mathrm{m}=\min \left\{\left|\mathrm{a}_{\mathrm{cc}}\right|, \mathrm{c}=1,2, \ldots, \mathrm{n}\right\}$ and radius is $\mathrm{S}_{\mathrm{te}}$. 


\section{Theorem 8 :}

$$
\begin{gathered}
\text { Suppose } A=\left(a_{c d}\right)_{n \times n} \in S_{I I I}(n), B=\left(b_{c d}\right)_{m \times m} \in S_{I I I}(m) \\
\text { and } m_{1}=\min \left\{\left|a_{c c}\right|, c=1,2, \ldots, n\right\} \text { and } \\
m_{1}^{\prime}=\max \left\{\left|\sum_{c=1}^{n} a_{c d} \cdot \sum_{d=1}^{n} a_{c d}\right|, c, d=1,2, \ldots, n\right\} \\
m_{2}=\min \left\{\left|b_{d d}\right|, d=1,2, \ldots, m\right\}, \\
m_{2}^{\prime}=\max \left\{\left|\sum_{c=1}^{n^{\prime}} b_{c d} \cdot \sum_{d=1}^{n} b_{c d}\right|, c, d=1,2, \ldots, n\right\} \\
\lambda(A \otimes B) \subset G(A \otimes B)=\left\{Z:\left|Z-a_{c c}\right| \leq \sqrt{(n-1) m_{2}\left(1-a_{c c}\right)}\right\} \cdot\left\{z:\left|Z-a_{d d}\right| \leq \sqrt{(n-1) m_{2}^{\prime}\left(1-a_{d d}\right)}\right\}
\end{gathered}
$$

Where $\lambda(A \otimes B)$ is denoted the whole eigen values of tensor for matrix $A$ and matrix $B, G(A \otimes B)$ is the oval region of the product for elements of balls whose center is $\mathrm{m}_{1}=\min \left\{\left|\mathrm{a}_{\mathrm{cc}}\right|, \mathrm{c}, \mathrm{d}=1,2, \ldots, \mathrm{n}\right\}$ and radius $\mathrm{S}+\mathrm{m}_{1}$ and balls whose center is $\mathrm{m}_{2}=\min \left\{\left|\mathrm{b}_{\mathrm{dd}}\right|, \mathrm{d}=1,2, \ldots, \mathrm{m}\right\}$ and radius $\mathrm{S}+\mathrm{m}_{2}$.

\section{Proof:}

$\lambda_{\min }(A \otimes B) \subset G_{\min }(A \otimes B)=\left\{Z:\left|z-a_{c c}\right| \leq \sqrt{(n-1) m_{1}\left(1-a_{c c}\right)}\right\} \cdot\left\{Z:\left|z-a_{d d}\right| \leq \sqrt{(n-1) m_{2}\left(1-a_{d d}\right)}\right\}$ $\lambda_{\max }(A \otimes B) \subset G_{\max }(A \otimes B)=\left\{Z:\left|z-\frac{\operatorname{Tra}(A \otimes B)}{n}\right| \leq \sqrt{\frac{n-1}{n} \sum_{c=1}^{n} \sum_{d=1}^{n} m_{1}^{\prime} \cdot m_{2}^{\prime}-\frac{(\operatorname{Tra}(A \otimes B))^{2}}{n}}\right\}$

Therefore, the maximum and minimum eigenvalues of tensor product for Matrix A and B. Hence proved.

\section{Theorem}

Suppose $A=\left(a_{c d}\right)_{n \times n} \in S(n), a_{c c}$ and $a_{d d}$ ar the most small module diagonal cross elements in $\mathrm{A}$, then $\lambda(\mathrm{A}) \subset \mathrm{G}(\mathrm{A})=\left\{\mathrm{Z}:\left|\mathrm{Z}-\mathrm{a}_{\mathrm{cc}}\right|\left|\mathrm{Z}-\mathrm{a}_{\mathrm{dd}}\right| \leq\left(\mathrm{S}+\left|\mathrm{a}_{\mathrm{cc}}\right|\right)\left(\mathrm{S}+\left|\mathrm{a}_{\mathrm{dd}}\right|\right)\right\}$ Where $\lambda(A)$ is denoted the whole eigen values of matrix $A$, $\mathrm{G}(\mathrm{A})$ is denoted cassini oval region of matrix $A$.

\section{Theorem}

Suppose

$$
\mathrm{A}=\left(\mathrm{a}_{\mathrm{cd}}\right)_{\mathrm{n} \times \mathrm{n}} \in \mathrm{S}_{\mathrm{III}}(\mathrm{n})
$$

and

$\mathrm{B}=\left(\mathrm{b}_{\mathrm{cd}}\right)_{\mathrm{m} \times \mathrm{m}} \in \mathrm{S}_{\mathrm{III}}(\mathrm{m})$ are quaternion doubly stochastic matrices, then

$$
\begin{gathered}
\lambda(\mathrm{A} \otimes \mathrm{B}) \subset \mathrm{G}(\mathrm{A} \otimes \mathrm{B})=\left\{\mathrm{Z}:\left|\mathrm{Z}-\mathrm{a}_{\mathrm{cc}}\right|\left|\mathrm{Z}-\mathrm{a}_{\mathrm{dd}}\right| \leq\left(\mathrm{S}+\left|\mathrm{a}_{\mathrm{cc}}\right|\right)\left(\mathrm{S}+\left|\mathrm{a}_{\mathrm{dd}}\right|\right)\right\} \\
\left\{\mathrm{Z}:\left|\mathrm{Z}-\mathrm{b}_{\mathrm{cc}}\right|\left|\mathrm{Z}-\mathrm{b}_{\mathrm{dd}}\right| \leq\left(\mathrm{S}+\left|\mathrm{b}_{\mathrm{cc}}\right|\right)\left(\mathrm{S}+\left|\mathrm{b}_{\mathrm{dd}}\right|\right)\right\}
\end{gathered}
$$

Where $\lambda(A \otimes B)$ is denoted the whole eigen values of tensor product for matrix $A$ and matrix $B, G(A \otimes B)$ is the oval region of the product for cassini oval region elements of matrix $\mathrm{A}$ and cassini oval region elements of matrix B.

\section{Conclusion}

In this paper is to locate and estimate the eigenvalues of quaternion doubly stochastic matrices then we present a several estimation theorems about the eigen values of quaternion doubly stochastic matrices. Finally, we obtain the eigenvalues using tensor product of two quaternion doubly stochastic matrices.

\section{References:}

[1] Brauer.A (1964). On the characteristic roots nonnegative matrices, Recent advances in Matrix Theory. Wiles Press. 3-38

[2] Gu, Yixi. (1994). The distribution of eigenvalues of matrix. Acta mathematics Applicatae Sinica, (04), 501511.

[3] Guo, Zhong. (1987). The existence of real double stochastic wit hpositive eigenvalue. Mathem,atics in practice and theory, (01).

[4] Lin, Lvuduan. (1984). Estimates of determinant and eigenvalue for two types matrices of row module equaling. Math ematics in practice and theory, (04).

[5] Shen, Guangxing. (1991). The Distribution of Eigenvalue of Generalized Stochastic Matrix. Chinese Journal of Engineering Mathematics, (03), 111-115.

[6] Tong, Wenting. (1997). Eigenvalue distribution on several types of matrices. Acta mathematics Sinica, (04). 